\title{
Application of Signal-Processing Techniques to Reduce the Errors Related to the FDTD Excitations
}

\author{
Levent Gürel and UĞur. OĞUZ*
}

DEPARTMENT OF ELECTRICAL AND ELECTRONICS ENGINEERINC BILKENT UNIVERSITYY

BilKENT, ANKARA, TURKEY

(lgurel@ee.bilkent.edu.tr, uoguz@cem.bilkent.edu.tr)

\section{Introduction}

The approximate nature of the FDT'D nethod causes the propagation of clectromagnetic waves in the conputational domain deviate from the ideal situation. The waves de not propagate with the theoretical phase velocities in the FDTD grid due to the nunerical dispersion errors.

The FDTD method requircs an initial state with all variables set to zero. If, for example, the physical system is simulated at $1 \mathrm{GHz}$, then the input is a $1-\mathrm{GHz}$, simusoidal multiplied by a step function, as displayed in Fig. 1(a). However, although its largest component is $1 \mathrm{GHz}$, the input contains many other frequency components, as demonstrated in Fig, 1(b). Because of aliasing and other numerical error sources, the transients due to these components may not completely vanish and the steady-state result may not be a perfect sinusoid. The amount of distortion varies with the sampling rate and the amplitudes of the high-frequency components fed into the system [1]. Increasing the sampling rates in space and time will certainly reduce the errors in general, but with the undesired disadvantage of increased computational cost. An alternative is to employ some signal-processing techniques to eliminate the errors due to the unnecessary high-frequency components.

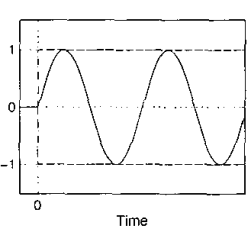

(a)

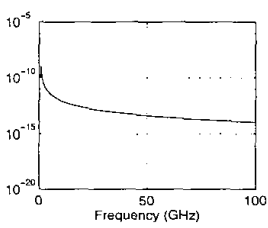

(b)

Figure 1: (a) Time-domain and (b) frequency-domain representations of a sinusoidal signal multiplied by a step function at $t=0$.

As an example, smoothing windows are used to taper the discontinuities of the signals and to reduce the high-frequency components of the signal spectra. For example, the sinusoidal signal in Fig. 1(a) is multiplied by a Hanning window of length $T_{0}$, where $T_{0}$ is one period of the sinusoidal signal. Figures 2(a) and (b) show the time- and frequency-domain respresentations of this windowed sinusoidal signal, respectively. Comparison of Fig. 2(b) to Fig. 1(b) demonstrates the decrease in the high-frequency components. 


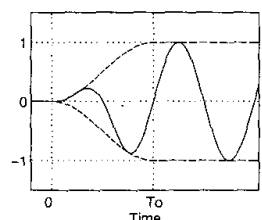

(a)

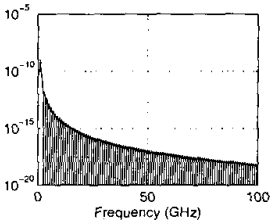

(b)

Figure 2: (a) Time-domain and (b) frequency-domain representations of a sinusoidal signal multiplied by a Hanning window with length $L=T_{0}$ at $t=0$.

\section{Homogeneous-Grid Simulation Results}

\subsection{Plane-Wave Excitation}

In this paper, total-field formulation with the 1-D look-up table will be used for the generation of the incident fields [2]. In this formulation, the absence of any scatterer implies the fields in the total-field region to be exactly the same as the incident wave and the fields in the scattered-field region to be exactly zero. However, computationally obtained fields deviate from their ideal counterparts. In order to quantify this deviation, the propagation of a $1-\mathrm{GHz}$ sinusoid in a homogeneous medium is simulated. The electric field is $z$ polarized and has unity amplitude. The magnetic field is polarized in the direction of $\hat{x}-\hat{y}$.

The maximum of the error in $E_{z}$ is computed at each time step and shown in Fig. 3(a). which demonstrates that the error decreases very slowly and does not reach its steady-state value after 800 time steps, almost 10 periods. Figure 3(b) displays the spectrum of the error, which is computed by the difference of the total-and incident-field components, in the $E_{z}$ component at the last cell of the total-field region. Figure 3 (b) demonstrates that the error is dominated by the components between $1 \mathrm{GHz}$ and $16.2 \mathrm{GHz}$.

Figure 4 displays the error results obtained with the sinusoid multiplied by a smoothing window. The smoothing is performed by a Hanning window of length $T_{0}$. Comparison of Fig. 3(a) to Fig. 4(a) reveals that the steady-state error level decreases when a smoothing is applied. When compared to Fig. 3(b), Fig. 4(b) demonstrates the decrease in almost all of the frequency components of the error signal by the application of the smoothing window.

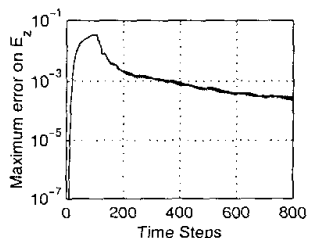

(a)

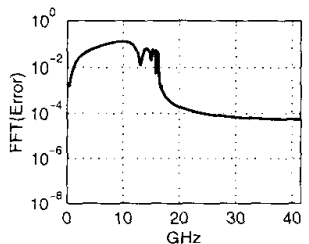

(b)

Figure 3: Error results obtained with no smoothing window. (a) Maximum crror on $E_{z}$. (b) Spectrum of the error on $E_{z}$ at the far corner of the total-field donnain. 


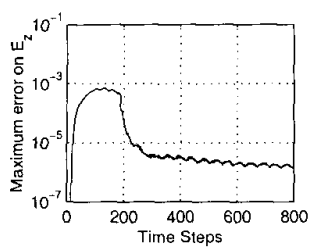

(a)

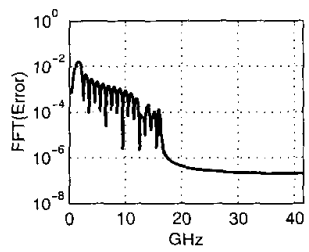

(b)

Figure 4: Error results obtained with Hanning window of length $L=T_{0}$. (a) Maximum error on $E_{z}$. (b) Spectrum of the error signal on $E_{z}$ at the far corner of the total-field domain

\subsection{Dipole Radiation}

In contrast to the plane-wave excitation scheme, there is no exact definition of error in the propagation of the waves produced by a finite source. However, the frequency-domain solution of an infinitesimal dipole in homogeneous space is analytically available. The infinitesimal dipole is modeled by a single Yee cell. The first input is a sinusoid multiplied by a step function, and the FD'TD observation is displayed in Fig. 5(a). The analytical solution is displayed in Fig. 5(c). Comparison of Fig. 5(a) to (c) reveals that the FDTD scheme cannot follow the abrupt change at $t=0$, and the oscillations are still observed after a number of periods of the pulse. These high-frequency components are visible in Fig. $5(b)$, the FFT of the signal in Fig. 5(a), around a band centered at $50 \mathrm{GHz}$. Multiplying the first period of the incident pulse by a smoothing window provides an FDTD signal identical to its theoretical counterpart, as demonstrated by Figs. 5(d) and (f). The suppression of the high-frequency components by the window is demonstrated by Fig. 5(e).

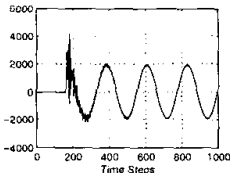

(a)

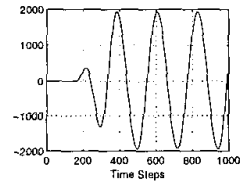

(d)

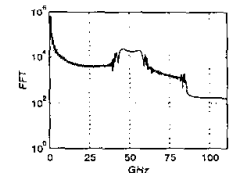

(b)

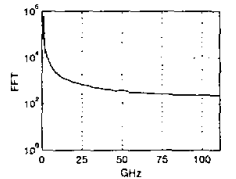

(e)

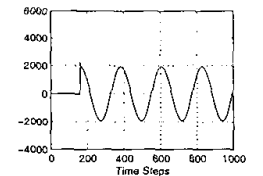

(c)

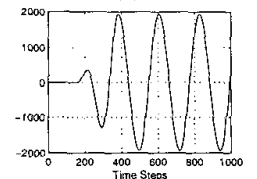

(f)

Figure 5: (a) FDTD result of a dipole excited by a causal sinusoid. (b) FFT of the signal in (a). (c) Analytical solution. (d) FDTD result of a dipole excited by a windowed sinusoid. (c) FFT of the signal in (d). (f) Analytical solution. 


\section{Simulation Results of Scattering Problems}

Plane-wave scattering problems involving conducting objects encounter a difficulty in the amplitude and phase detection on the signals at observation points located close to the scatterer. The DC offsets on the surface current signals do not decay or decay very slowly with time [3]. Such a case is demonstrated by simulating the scattered fields from a conducting prism of $20 \times 20 \times 100$ cells illuminated by a $z$-polarized plane wave of $4 \mathrm{GHz}$, incident from the $+y$ direction. The simulation parameters of this 3-D problem are similar to those of the 2-D problem reported in [3]. The observation point is locaterl at 4 cells from the bright face of the scatteres. The time dependence of the plane wave is a simusoid multiplied by a step function. The simulation restlt, displayed in Fig. 6(a), exhibits a visible DC offset value? even after five periods of the incident wave. This DC value does not fall uncler $1 \%$ of the amplitude of the field variable after 16 pexiods of the incident wave.

In the next simulation, the sinusoidal source is multiplied by a Hanning window of half-period length. The same magnetic-field component, plotted in Fig. 6(b), rapidly reaches the steady state with almost no $\mathrm{DC}$ value. After three and a half periods of the incident wave, the $d c$ value is below $1 \%$ of the amplitude value and goes on decreasing down to $0.1 \%$ after 16 periods. Although both simulations rcach the steady state, the source function with lower high-frequency content requires much less computation time to deliver the accurate results.

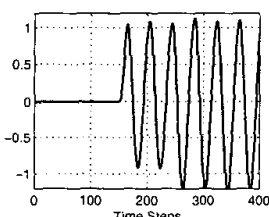

(a)

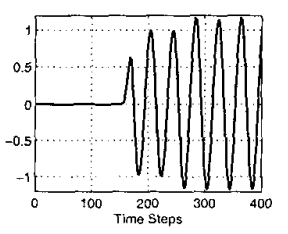

(b)

Figure 6: The normalized $H_{x}$ components observed 4 cells away from the face of a conducting prism. The incident plane wave is a sinusoid multiplied by a (a) step function, (b) one-period-long Hanning window.

\section{Concluding Remarks}

The signal-processing tool smoothing window is used to enhance the excitation scheme of the FDTD method. The improvements in both plane-wave and finitesource excitation schemes are demonstrated. Applications of other signal-processing techniques will also be demonstrated in the talk.

\section{References}

[1] L. Gürel and U. Oğuz, "Signal-processing techniques to reduce the sinusoidal steadystate error in the FDTD method," IEEE Trans. Antennas Propagat., vol. 48, no. 4, pp. 585-593, Apr. 2000.

[2] A. Taflove, Computational Electrodynarnics: The Finite-Difference Time-Domain Method, Artech House, Boston, MA, 1995.

[3] C. M. Furse, S. P. Mathur, and O. P. Gandhi, "Improvements to the finite-difference time-domain method for calculating the radar cross section of a perfectly conducting target," IEEE Trans. Microwave Theory Tech., vol. 38, no. 7, pp. 919-927, July 1990. 